\title{
DIVERSIDAD Y ESTRUCTURA GENÉTICA DE ARTIBEUS JAMAICENSIS (CHIROPTERA: PHYLLOSTOMIDAE) EN CHIAPAS, MÉXICO
}

\author{
GENETIC DIVERSITY AND STRUCTURE OF ARTIBEUS JAMAICENSIS \\ (CHIROPTERA: PHYLLOSTOMIDAE) IN CHIAPAS, MEXICO
}

\section{VIRIDIANA LLAVEN MACÍAS, ${ }^{1}$ LORENA RUIZ MONTOYA, ${ }^{1}$ MARICELA GARCÍA BAUTISTA, ${ }^{1}$ JULIA LESHER GORDILLO ${ }^{3}$ y SALIMA MACHKOUR M'RABET ${ }^{2, *}$}

\author{
${ }^{1}$ El Colegio de la Frontera Sur. Departamento de Conservación de la Biodiversidad. Unidad San Cristóbal de \\ las Casas. Carretera Panamericana s/n. Barrio de María Auxiliadora, C.P. 29200, San Cristóbal de Las Casas, \\ Chiapas, México.<vllaven-m@hotmail.com>, <lruiz@ecosur.mx>, <mgarcia@ecosur.mx>. \\ ${ }^{2}$ El Colegio de la Frontera Sur. Departamento de Conservación de la Biodiversidad. Unidad Chetumal. Av. \\ Centenario Km 5.5, C.P. 77014, Chetumal, Quintana Roo, México. <smachkou@ecosur.mx> \\ ${ }^{3}$ Universidad Juárez Autónoma de Tabasco. División Académica de Ciencias Biológicas (DACBiol). Carretera \\ Villahermosa-Cárdenas Km. 0.5 S/N, Entronque a Bosques de Saloya. C.P. 86150. Villahermosa, Tabasco, \\ México.<lesher23@yahoo.com>. \\ * Autor de correspondencia: <smachkou@ecosur.mx> \\ Recibido: 25/05/2016; aceptado: 07/11/2016 \\ Editor responsable: Vinicio Sosa
}

Llaven-Macías, V., Ruiz-Montoya, L., García-Bautista, M., Lesher-Gordillo, J. y Machkour-M'Rabet, S. (2017) Diversidad y estructura genética de Artibeus jamaicensis (Chiroptera: Phyllostomidae) en Chiapas, México. Acta Zoológica Mexicana (n.s.), 33(1), 55-66.

RESUMEN. La deforestación de las selvas provoca la fragmentación de los hábitats, lo cual interviene en la composición, abundancia y demografía de las especies, y por lo tanto, en el aislamiento poblacional en muchas especies. Los impactos de la fragmentación dependerán de la capacidad de respuesta de las especies a los disturbios en su hábitat, y de la configuración y estructura del paisaje. En algunas poblaciones, la fragmentación del hábitat puede provocar el aislamiento poblacional a largo plazo, por la reducción del tamaño poblacional y el debilitamiento de relaciones entre individuos, aún en especies de alta movilidad como los murciélagos. En México, A. jamaicensis es de los murciélagos más estudiados; sin embargo, son pocos los trabajos enfocados en conocer los impactos de la modificación de su hábitat, en su diversidad y estructura genética. Por lo tanto, el objetivo de este trabajo fue evaluar la diversidad, la estructura y la diferenciación genética, así como analizar la historia demográfica de $A$. jamaicensis en dos hábitats diferentes, utilizando un fragmento de 396 pb de la región mitocondrial D-loop. La red de haplotipos reveló 34 haplotipos únicos de 34 individuos analizados. La diversidad haplotípica fue alta $(h=1)$ para ambas poblaciones, y la diversidad nucleotídica fue relativamente baja $(<0.03)$. El análisis de la distribución de diferencias nucleotídicas pareadas (distribución mismatch), y los valores negativos de los estadísticos basados en pruebas de neutralidad, sugieren un proceso de expansión demográfica reciente y repentina de A. jamaicensis. Una moderada diferenciación genética apunta a que existe estructura genética de $A$. jamaicensis.

Palabras clave: D-loop, diversidad genética, estructura, haplotipos, historia demográfica.
Llaven-Macías, V., Ruiz-Montoya, L., García-Bautista, M., Lesher-Gordillo, J., \& Machkour-M'Rabet, S. (2017) Genetic diversity and structure of Artibeus jamaicensis (Chiroptera: Phyllostomidae) in Chiapas, México. Acta Zoológica Mexicana (n.s.), 33(1), 55-66.

ABSTRACT. Deforestation causes fragmentation of habitats, which in turn affects the composition, abundance and demography of species, and therefore the population isolation of many species. The impacts of fragmentation will depend on the responsiveness of the species to disturbances in their habitat, and the configuration and structure of landscape. In some populations, habitat fragmentation can cause long-term population isolation by the reduction in population size and the weakening of relations between individuals, even in highly mobile species like bats. In Mexico, A. jamaicensis is the most studied bat; however, few studies focused on the knowledge of the impacts of habitat modification in its diversity and genetic structure. Therefore, the objective of this study was to evaluate the diversity, structure and genetic differentiation, and analyze demographic history of A. jamaicensis in two different habitats, using a $396 \mathrm{bp}$ fragment of the mitochondrial Dloop region. Haplotype network revealed 34 unique haplotypes of 34 individuals analyzed. Haplotype diversity was high $(h=1)$ for both populations, and nucleotide diversity was relatively low $(<0.03)$. The analysis of the distribution of pairwise differences between sequences and negative values of statistical tests based on neutrality suggest a process of recent and sudden demographic expansion of $A$. jamaicensis. A moderate genetic differentiation points to the existence of genetic structure of $A$. jamaicensis.

Key words: demographic history, D-loop, genetic diversity, haplotypes, structure. 


\section{INTRODUCCIÓN}

México se ubica entre los cinco países considerados megadiversos por su alta diversidad de especies, que representa aproximadamente el 12\% del total mundial (Mittermeier et al. 1997). Sin embargo, la biodiversidad se ha visto amenazada por la deforestación de bosques y selvas, producto del cambio de uso de suelo con fines pecuarios y agrícolas, así como para el desarrollo urbano (FAO 2010). $\mathrm{Al}$ deforestarse grandes extensiones de selvas y bosques, se provoca la fragmentación o pérdida de hábitats de muchas especies (Wilcox \& Murphy 1985). En este sentido, se entiende por fragmentación al proceso de división de un hábitat en una serie de parches más pequeños, aislados unos de otros (Wilcove et al. 1986), donde el grado de la fragmentación determina el tamaño y el aislamiento de los parches o fragmentos (Fahrig 2003).

La fragmentación o pérdida de hábitats tiene grandes efectos negativos, no solo en la riqueza y composición de especies (Wilcove et al. 1986; Fahrig 2003), pero también en la abundancia y distribución de muchas especies (Fahrig 2003). La magnitud de estos efectos dependerá del tiempo del aislamiento del fragmento, de la configuración y la estructura del paisaje, de la distancia y el grado de conectividad entre los fragmentos, del tamaño y forma del fragmento, de la posición de los fragmentos en el paisaje (Saunders et al. 1991), y de las características específicas de cada especie (Bicca-Marques 2003), que les permitirá su permanencia o extinción en el paisaje (Galindo-González 2007). Por ejemplo, los efectos de la fragmentación sobre las comunidades de murciélagos son la pérdida de especies con requerimientos especializados de hábitat, debido a que no pueden salir de los remanentes del bosque, y un incremento en la abundancia de especies generalistas, por su alta capacidad de desplazamiento en todo el paisaje (Galindo-González 2004; Galindo-González 2007; Sánchez 2009).

La fragmentación del hábitat también puede provocar el aislamiento poblacional a largo plazo, por la reducción en el tamaño poblacional y el debilitamiento de relaciones entre individuos (Kunz et al. 2007; Sánchez 2009), generando discontinuidades en el flujo génico entre poblaciones, e impactando en la estructura y diversidad genética (Holderegger \& Di Giulio 2010). El aislamiento poblacional dependerá de la capacidad de respuesta de las especies a los disturbios en su hábitat (Galindo-González 2004). Por ejemplo, algunos murciélagos filostómidos frugívoros (subfamilias Carollinae y Stenodermatinae), frecuentemente aumentan su abundancia en los bosques perturbados (Fenton et al. 1992; Galindo-González 2004; Peters et al. 2006), pero son severamente afectados cuando ocurren cambios drásticos en sus refugios y sitios de forrajeo (Jones et al. 2001; Soriano \& Ochoa 2001). Sin embargo, el murciélago zapotero Artibeus jamaicensis Leach, 1821 (subfamilia Stenodermatinae), bajo condiciones de perturbación del hábitat, puede mostrar una alta selectividad por el bosque tropical y baja abundancia en hábitats perturbados (Castro-Luna 2007). Así mismo, el huracán Georges (Puerto Rico, 1998) ocasionó el decline poblacional de $A$. jamaicensis, debido no solo a la muerte de muchos individuos por los fuertes vientos, sino también a la destrucción de sus refugios y sitios de forrajeo (Jones et al. 2001).

Artibeus jamaicensis es una de las especies más abundantes de murciélagos neotropicales (Ortega \& Arita 1999), de amplia distribución geográfica en el continente americano (Eisenberg \& Redford 1999), con alta movilidad (vuelo de larga distancia), se adapta fácilmente a cualquier tipo de hábitat y ocupa una amplia variedad de refugios (Ortega \& Castro-Arellano 2001; Larsen et al. 2007). Es esencialmente frugívoro, y se alimenta de una amplia variedad de especies vegetales (Studier et al. 1983); pero si estos recursos son escasos puede consumir néctar, insectos y polen (Ortega \& Castro-Arellano 2001). Lo anterior permite que generalmente $A$. jamaicensis no se vea afectado por la perturbación y fragmentación de sus hábitats, lo cual se refleja en su alta diversidad genética y alto flujo génico (Carstens et al. 2004; VázquezDomínguez et al. 2013).

Sin embargo, se han reportado poblaciones de $A$. jamaicensis con alta diferenciación genética dentro y entre ellas, a una escala geográfica fina (Landaverde-González et al. 2012). La escala geográfica juega un papel muy importante en el aislamiento poblacional, principalmente en murciélagos que pueden volar kilómetros de distancia entre fragmentos de vegetación (Rossiter et al. 2000), como A. jamaicensis. A pesar de la alta vagilidad de algunas especies de murciélagos, es posible encontrar alta diferenciación genética cuando se evalúan a gran escala geográfica (Miller-Butterworth et al. 2003; Juste et al. 2009).

Los murciélagos son considerados altamente móviles $\mathrm{y}$, por lo tanto, son menos susceptibles a la fragmentación del hábitat que otros animales (Ripperger et al. 2013). Sin embargo, la capacidad de movilidad de cada especie determina su vulnerabilidad a la fragmentación de su hábitat, y su estructura genética (Meyer et al. 2009). Ripperger et al. (2013) estudiaron a escala geográfica fina (de aproxi- 
madamente $20 \mathrm{~km}$ ), poblaciones de Artibeus watsoni Thomas, 1901 (Chiroptera: Phyllostomidae), y encontraron correlación de la diversidad genética con el tamaño de los parches. Así mismo, Carollia castanea Allen, 1890 (Chiroptera: Phyllostomidae) responde de mejor manera a la fragmentación del hábitat a pequeña escala que otras especies de murciélagos filostómidos, y su flujo génico disminuye a menudo que aumenta la distancia geográfica (Ripperger et al. 2014). Ambas especies, son frugívoras y comunes en bosques primarios y secundarios (Melo et al. 2009; Reid 2009).

En general, A. jamaicensis es de los filostómidos más estudiados a nivel molecular, con estudios enfocados en la filogenia (Lim et al. 2004), la filogeografía de especies (Phillips et al. 1991; Larsen et al. 2007; Fleming et al. 2009), y la genética de poblaciones (Pumo et al. 1996; Carstens et al. 2004; Landaverde-González et al. 2012). En México, A. jamaicensis también ha sido de los murciélagos más estudiados. En este sentido, los trabajos han mostrado alta diversidad genética y nula estructura poblacional de A. jamaicensis (Vargas-Miranda 2009; Vázquez-Domínguez et al. 2013); mientras Ortega et al. (2003) analizaron las relaciones entre machos dominantes y subordinados, y encontraron una relación de parentesco padre-hijo, y tampoco encontraron estructura poblacional. Ruiz et al. (2013) analizaron poblaciones de A. jamaicensis distribuidas desde América Central hasta el oeste de la península de Yucatán, de las cuales encontraron dos haplogrupos con una amplia diversidad genética y fuerte estructura genética. Los análisis de la historia demográfica de los dos haplogrupos, señalan que ambos sufrieron eventos sucesivos de expansión poblacional.

Aunque, A. jamaicensis ha sido de los murciélagos más estudiados genéticamente en México, solo un trabajo se ha enfocado en entender el efecto de las características ecológicas del hábitat sobre la diversidad y estructura genética de A. jamaicensis (Vázquez-Domínguez et al. 2013). Por lo tanto, es importante ampliar esta información, debido a que la diversidad genética de poblaciones locales de A. jamaicensis podría verse afectada por la modificación y fragmentación del hábitat. En el estado de Chiapas (México) la deforestación debida a la ganadería es muy frecuente (Covaleda et al. 2014), lo que podría afectar las poblaciones de murciélagos. Sin embargo, no se han realizado estudios genéticos de $A$. jamaicensis, para evaluar el impacto de diferentes hábitats en las poblaciones de A. jamaicensis. Por lo tanto, los objetivos de esta investigación fueron: (I) evaluar la diversidad genética, (II) determinar la estructura y la diferenciación genética, y (III) evaluar la historia demográfica de $A$. jamaicensis en dos hábitats diferentes en Chiapas.

\section{MATERIAL Y MÉTODOS}

Área de estudio. Los individuos de A. jamaicensis se capturaron en dos localidades de selva mediana subperennifolia en la región fisiográfica Llanura Costera del Pacífico, Chiapas, con una distancia entre ellas de $29 \mathrm{~km}$ (Fig. 1). Se consideraron a priori como dos poblaciones por la distancia y condiciones contrastantes del hábitat. La primera localidad se conoce como Pantaleón (Mpio. de Mapastepec; 15³1'33.0” N - 9249'21.6” W; 585 $\mathrm{msnm}$ ), este sitio comprende vegetación densa y nativa, en la cual se observó la presencia de especies raras de murciélagos, vulnerables a la perturbación de su hábitat, y reportadas en bosques maduros y densos. La segunda localidad es Manacal (Mpio. de Pijijiapan; 15³8'24.8"

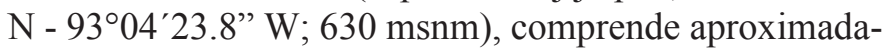
mente 8 ha de vegetación modificada (selva con platanar, árboles ornamentales y comestibles) y rodeada de grandes extensiones de potreros.

Colecta de muestras. Se utilizaron dos redes de niebla de $2 \times 12$ m colocadas entre senderos de las 18:00 a 24:00 horas y revisadas cada media hora. Los individuos de $A$. jamaicensis se identificaron con base a la clave de identificación de Medellín et al. (2008). Se capturaron 34 individuos de $A$. jamaicensis en abril de 2015: 19 individuos en Manacal y 15 individuos en Pantaleón. El ADN de $A$. jamaicensis se obtuvo de una pequeña porción de la membrana alar, cortando aproximadamente $3 \mathrm{~mm}$ del uropatagio con un bisturí estéril. Las muestras se colocaron en viales de $1.5 \mathrm{ml}$ con alcohol al 96\% hasta su posterior análisis genético.

Extracción, amplificación y secuenciación de ADN. La extracción de ADN se realizó siguiendo el protocolo de Fenol-Cloroformo-Alcohol isoamílico (Sambrook et al. 1989). Se amplificó un fragmento de ADN de la región mitocondrial D-loop con los iniciadores E (5'-CCT GAAGTA GGA ACC AGA TG-3'; Wilkinson \& Chapman 1991) y P (5'-CCC CAC CAT CAA CAC CCA AAGCTGA-3'; Wilkinson et al. 1997). En cada amplificación se utilizó la técnica de reacción en cadena de la polimerasa (PCR), con un volumen total de $39 \mu 1$ constituido por: 18 $\mu 1$ de mezcla maestra (Mastermix, PROMEGA), $15 \mu 1$ de agua ultrapura, $2 \mu 1$ iniciador E, $2 \mu 1$ iniciador $\mathrm{P}$, y $2 \mu 1$ de ADN molde. Las amplificaciones se realizaron en un termociclador de gradiente MJ Mini (BIO-RAD) con el 


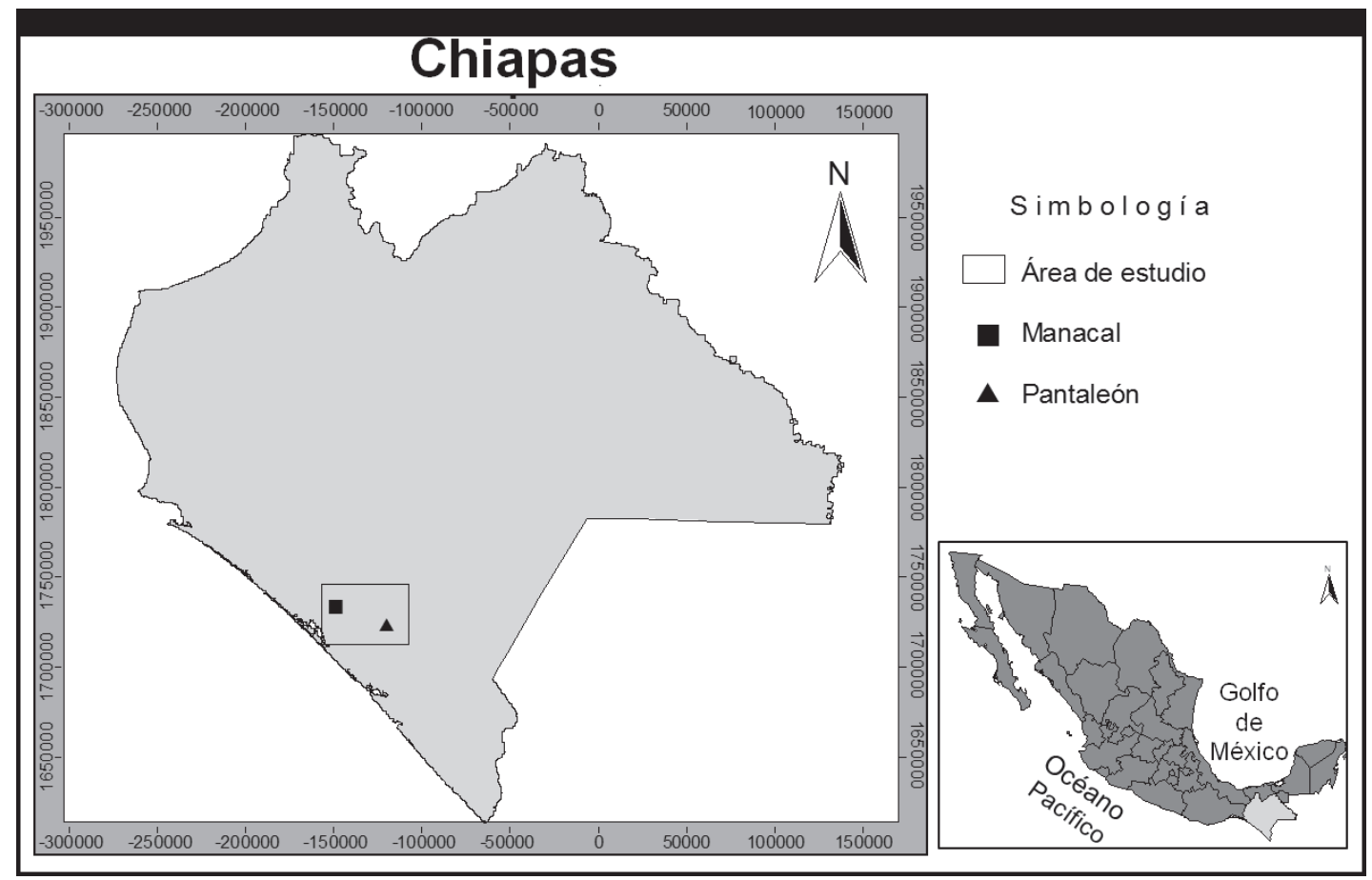

Figura 1. Área de muestreo de Artibeus jamaicensis, en dos hábitats contrastantes en Chiapas, México.

siguiente programa: ciclo inicial de $94{ }^{\circ} \mathrm{C}$ por 4 minutos, seguidos de 35 ciclos de $94{ }^{\circ} \mathrm{C}$ por 60 segundos, $55^{\circ} \mathrm{C}$ por 90 segundos, $72{ }^{\circ} \mathrm{C}$ por 60 segundos, y un ciclo final de $72{ }^{\circ} \mathrm{C}$ por 10 minutos. El producto de las reacciones de PCR fueron visualizadas en electroforesis $(100 \mathrm{~V} ; 50$ minutos), en gel de agarosa al $2 \%$ en medio TAE $1 \mathrm{X}$ con tinción de Bromuro de Etidio ( $1 \mathrm{~g} / 100 \mathrm{ml}$ en TAE $1 \mathrm{X})$, con un marcador control de $100 \mathrm{pb}$ (DNA ladder, PROMEGA). La purificación y secuenciación por electroforesis capilar (Sanger) de los productos de PCR se realizaron en la empresa Macrogen (Seúl, Corea del Sur).

Alineamiento y análisis de secuencias. Las 34 secuencias de $A$. jamaicensis se compararon con secuencias depositadas en el banco de genes (GenBank) para confirmar la identificación de la especie, con la ayuda de BLAST (Basic Local Alignment Search Tool; Altschul et al. 1990) de la base de datos NCBI (National Center for Biotechnology Information). Las secuencias y sus electroferogramas fueron revisadas y corregidas manualmente con el programa ChromasPro versión 1.5 (Technelysium Pty Ltd 2003-2009). Su alineación se realizó en ClustalX versión 2.0.11 (Larkin et al. 2007) con los parámetros por defecto.

Diversidad genética. La diversidad genética total y por localidad se estimó con la diversidad haplotípica $(\dot{h}=$ pro- babilidad de que dos haplotipos seleccionados al azar de una muestra sean diferentes) y la diversidad nucleotídica ( $\pi=$ número promedio de diferencias nucleotídicas por sitio entre dos secuencias escogidas al azar; Nei 1987), usando el programa DnaSP versión 5 (Librado \& Roza 2009). También se estimaron por localidad y en total, el número de sitios segregados o sitios polimórficos $(S)$, el número de transiciones y transversiones observadas, el número de sustituciones sinónimas por sitio (ks), el número promedio de las diferencias nucleotídicas entre pares de secuencias $(K)$ y la composición nucleotídica. Todos estos parámetros se determinaron con el programa Arlequín versión 3.1 (Excoffier et al. 2006).

Diferenciación y estructura genética. La diferenciación genética se estimó con los estadísticos de Hudson et al. (1992) y el índice de fijación $F_{S T}$ (Wright 1951). Los primeros se basan en el valor de $H_{S T}=1-\left(H_{\mathrm{S}} / H_{T}\right)$, donde $H_{\mathrm{S}}$ es el promedio ponderado de la diversidad haplotípica de las subpoblaciones y $H_{T}$ es la estimación de la diversidad haplotípica de la población total. Adicionalmente, se estimó el valor de $K_{S T}=1-\left(K_{S} / K_{T}\right)$, donde $K_{S}$ es el promedio ponderado de $\mathrm{K}_{1}$ y $\mathrm{K}_{2}$ (número promedio de las diferencias entre las secuencias en subpoblaciones 1 y 2 , respectivamente) y $K_{T}$ es el promedio de las diferencias entre dos secuencias de la muestra total (Hudson et al. 
1992). La significancia de los estadísticos de Hudson et al. (1992) se evaluó con la Chi-cuadrada y con 1,000 repeticiones en el programa DnaSP versión 5 (Librado \& Rozas 2009), y el $F_{S T}$ con 10,000 repeticiones en el programa Arlequín 3.1 (Excoffier et al. 2006). Para determinar cómo se encuentra repartida la variación genética en distintos niveles jerárquicos (entre y dentro poblaciones), se realizó un análisis de varianza molecular (AMOVA) con 10,000 repeticiones para las pruebas de significancia e intervalo de confianza al $95 \%$, en el programa Arlequín 3.1 (Excoffier et al. 2006).

Historia demográfica. Se examinó la historia demográfica de $A$. jamaicensis por localidad y en total, con base en la distribución de diferencias nucleotídicas pareadas (distribución mismatch) (Slatkin \& Hudson 1991) y el modelo de neutralidad. El primero se basa en tres parámetros: teta inicial $\left(\theta_{0}=\right.$ tamaño poblacional antes del proceso de expansión), teta final $\left(\theta_{1}=\right.$ tamaño poblacional después del proceso de expansión) y $\tau$ (tau $=$ tiempo de expansión en unidades de mutaciones) (Rogers \& Harpending 1992). La distribución mismatch multimodal indica equilibrio demográfico, y la unimodal una expansión demográfica poblacional relativamente reciente (Slatkin \& Hudson 1991; Rogers \& Harpending 1992). La significancia estadística se evaluó con 10,000 repeticiones, bajo la hipótesis nula del modelo de expansión demográfica repentina reciente, mediante la suma de desviaciones al cuadrado (SSD; por sus siglas en inglés) en el programa Arlequín versión 3.1 (Excoffier et al. 2006), y el estadístico $R_{2}$ (Ramos-Onsins $\&$ Rozas 2002) en el programa DnaSP versión 5 (Librado \& Rozas 2009).

El modelo de neutralidad tiene como hipótesis nula que la variación molecular es el resultado de un balance entre las mutaciones neutrales o al azar, y la extinción de mutaciones por deriva génica, manteniendo las frecuencias génicas en equilibrio (Kimura 1991; Perfectti et al. 2009). Para buscar desviaciones en este modelo se utilizaron los estadísticos D (Tajima 1989), D* (Fu \& Li 1993) y $F_{\mathrm{S}}$ (Fu 1997). Los valores negativos de estos estadísticos son esperados en poblaciones que han experimentado una expansión demográfica poblacional relativamente reciente o efecto de selección purificadora. Los valores positivos indican procesos de selección balanceadora (o equilibrada) o reducción del tamaño poblacional (Kaplan et al. 1989; Tajima 1989). El nivel de significancia estadística de las tres estimaciones se evaluó con 10,000 repeticiones (con simulación coalescente) en el programa DnaSP versión 5 (Librado \& Rozas 2009).

Análisis genealógicos. Las relaciones a nivel intraespecífico de $A$. jamaicensis se analizaron a partir de la construcción de una red de haplotipos, a través del método de Median-Joining de acuerdo al criterio de Bandelt et al. (1999) y máxima parsimonia, implementado en el programa Network versión 4.6.1.3. (Fluxus Technology Ltd 2012). Los parámetros empleados fueron: épsilon de 0 , peso transiciones- transversiones $1 / 1$, peso de caracteres $5 / 10$ y el criterio de costo de conexión.

\section{RESULTADOS}

Se analizaron 34 individuos de A. jamaicensis (18 machos y 16 hembras): 19 individuos (9 machos y 10 hembras) de Manacal y 15 individuos ( 9 machos y 6 hembras) de Pantaleón. El alineamiento de las secuencias arrojó una matriz de 396 (bp) por secuencia, incluyendo los eventos de inserción-delección y los huecos producidos por el alineamiento (gaps). El análisis de los 396 pb reveló 62 sitios polimórficos y 34 haplotipos únicos.

Diversidad genética. La diversidad haplotípica $(h)$ en ambas poblaciones tuvo un valor de 1 (Cuadro 1). La población Pantaleón presentó el mayor número de sustituciones y sitios segregados, lo que originó un valor de $\pi=0.0 .0293$, ligeramente mayor que el observado en

Cuadro 1. Diversidad genética por localidad, y en total, de Artibeus jamaicensis provenientes de dos hábitats contrastantes en Chiapas (México), basada en un segmento de 396 bp del D-loop. Las abreviaturas son: tamaño de la muestra (n), número de haplotipos (H), diversidad haplotípica $(h)$, diversidad nucleotídica $(\pi)$, número de transiciones observadas (Tras), número de transversiones observadas (Trv), número de sustituciones sinónimas $(\mathrm{ks})$, número de sitios polimórficos o número de segregados $(S)$, número promedio de las diferencias nucleotídicas $(K)$, composición nucleotídica $(\mathrm{C}=$ Citocina, $\mathrm{T}=$ Timina, $\mathrm{A}=$ Adenina, $\mathrm{G}=$ Guanina $)$, y desviación estándar $(\mathrm{SD})$.

\begin{tabular}{|c|c|c|c|c|c|c|c|c|c|c|c|c|}
\hline \multirow[t]{2}{*}{ Poblaciones } & \multirow[t]{2}{*}{$\mathrm{n} / \mathrm{H}$} & \multirow[t]{2}{*}{$h(\mathrm{SD})$} & \multirow[t]{2}{*}{$\pi(\mathrm{SD})$} & \multirow[t]{2}{*}{ Tras } & \multirow[t]{2}{*}{ Trv } & \multirow[t]{2}{*}{ ks } & \multirow[t]{2}{*}{$S$} & \multirow[t]{2}{*}{$K(\mathrm{SD})$} & \multicolumn{4}{|c|}{ Composición nucleotídica (\%) } \\
\hline & & & & & & & & & $\mathrm{C}$ & $\mathrm{T}$ & A & $\mathrm{G}$ \\
\hline Pantaleón & 15 & $1.0(0.024)$ & $0.0293(0.003)$ & 23 & 23 & 46 & 52 & $13.04(6.98)$ & 11.9 & 34.8 & 33.7 & 19.5 \\
\hline Total & 34 & $1.0(0.025)$ & $0.0249(0.002)$ & 31 & 24 & 55 & 62 & $11.39(5.30)$ & 11.7 & 34.8 & 33.6 & 19.8 \\
\hline
\end{tabular}


Manacal ( $\pi=0.0202$; Cuadro 1). La composición nucleotídica total y en ambas localidades, estuvo representada mayormente por Timinas (T) y Adeninas (A; Cuadro 1).

Diferenciación y estructura genética. De acuerdo con los estadísticos de Hudson et al. (1992) las poblaciones de Pantaleón y Manacal no presentaron diferenciación genética y subdivisión $\left(H_{\mathrm{ST}}=0.00 ; \chi^{2}=34.0, P=0.42\right.$, g.l. = 33). Sin embargo, basadas en las diferencias nucleotídicas, se pudo observar diferenciación genética significativa, con los dos tipos de parámetros determinados, los parámetros de Hudson et al. (1992) $\left(K_{\mathrm{S}}=9.33, K_{\mathrm{ST}}=\right.$ $0.03 ; P=0.019)$, y el índice de fijación $\left(F_{S T}=0.054 ; P=\right.$ 0.00079 ). La prueba de AMOVA (para 62 sitios polimórficos) reveló una ligera diferenciación con el $94.62 \%$ de la variación genética repartida entre individuos dentro de las localidades, mientras que el $5.38 \%$ se debe a variaciones entre las localidades (Cuadro 2).

Historia demográfica. La distribución mismatch, con varios picos ligeramente marcados, difiere de lo esperado bajo el modelo de expansión poblacional repentina, dando lugar a una distribución multimodal (Fig. 2). Sin embargo, la desviación no fue significativa en Pantaleón (SSD $=0.0106 ; P=0.39)$, Manacal $(\mathrm{SSD}=0.005 ; P=0.64)$ y en total $(\mathrm{SSD}=0.001 ; P=0.90)$. Así mismo, $R_{2}$ no es estadísticamente significativo para Pantaleón $(P=0.06)$, Manacal $(P=0.08)$ y en total $(P=0.13)$. Los valores negativos de los estadísticos $\mathrm{D}$ y $\mathrm{D} *$ no son estadísticamente significativos $(P>0.10)$. No obstante, el estadístico $F_{\mathrm{S}}$ es significativamente negativo $(P<0.02)$ en ambas localidades y en total (Cuadro 3).
Análisis genealógicos. Los valores de parsimonia obtenidos para la reconstrucción de la red de haplotipos fueron altos en todos los pasos $(P>0.95)$. Se identificaron 34 haplotipos únicos, los cuales se conectaron entre sí parsimoniosamente entre 1 y 9 pasos mutacionales (Fig. 3). No se observaron haplotipos agrupados de acuerdo a la ubicación geográfica, los haplotipos de Pantaleón se mezclan con los de Manacal.

\section{DISCUSIÓN}

Artibeus jamaicensis se adapta fácilmente a diferentes tipos de hábitat, posee una alta movilidad y alta abundancia, lo cual le facilita un flujo génico alto entre sus poblaciones (Rossiter et al. 2012; Vázquez-Domínguez et al. 2013). Estos aspectos de la historia natural de $A$. jamaicensis dan lugar a los altos niveles de diversidad genética, y la baja diferenciación genética encontrados en las poblaciones de Pantaleón y Manacal. Además, la distancia geográfica entre los dos hábitats podría no ser suficiente para aislar las poblaciones de A. jamaicensis, por su alta capacidad de vuelo.

Los valores de diversidad genética observados en la presente investigación son consistentes con lo reportado en estudios moleculares basados en el ADNmt de $A$. jamaicensis, los cuales señalan que esta especie posee una alta diversidad genética con valores de $h$ cercanos a 1.0 (Carstens et al. 2004; Ruíz et al. 2013; Del Real-Monroy et al. 2014). El impacto de la fragmentación del hábitat

Cuadro 2. Análisis de Varianza Molecular (AMOVA) de 34 secuencias de Artibeus jamaicensis de dos hábitats contrastantes en Chiapas (México), realizado a través de 10,000 repeticiones $(P<0.01)$ en el programa Arlequín 3.1 (Excoffier et al. 2006).

\begin{tabular}{lccc}
\hline Fuente de variación & Suma de cuadrados & Componentes de la Varianza & \% de variación \\
\hline Entre las poblaciones & 10.815 & 0.31479 & 5.38 \\
Dentro de las poblaciones & 177.214 & 5.53794 & 94.62 \\
Total & 188.029 & 5.85273 & 100 \\
\hline
\end{tabular}

Cuadro 3. Pruebas estadísticas de la distribución de las diferencias pareadas (distribución mismatch) e índices de neutralidad de Artibeus jamaicensis de dos hábitats contrastantes en Chiapas (México), basadas en un segmento de 396 pb de la región mitocondrial D-loop: tamaño de la población $(\mathrm{N})$, teta inicial $\left(\theta_{0}=2 \mu \mathrm{N} 0\right)$, teta final $\left(\theta_{1}=\mu \mathrm{N} 1\right)$, tau $(\tau=2 \mu \mathrm{t})$, estadístico $R_{2}$ (Ramos-Onsins \& Rozas 2002), estadístico D (Tajima 1989), estadístico $D^{*}$ (Fu \& Li 1993) y estadístico $F_{\mathrm{S}}(\mathrm{Fu} 1997)$. Nivel de significancia estadística: ${ }^{\text {ns }} P>0.05$ (no significativo); $* P \leq 0.05$ (significativo).

\begin{tabular}{lcccccccc}
\hline Poblaciones & $\mathrm{N}$ & $\theta_{0}$ & $\theta_{1}$ & $\tau$ & $R_{2}$ & $\mathrm{D}$ & $\mathrm{D}^{*}$ & $F_{\mathrm{S}}$ \\
\hline Pantaleón & 15 & 1.814 & 1000 & 9.576 & $0.096^{\mathrm{ns}}$ & $-0.759^{\mathrm{ns}}$ & $-1.136^{\mathrm{ns}}$ & $-6.07^{*}$ \\
Manacal & 19 & 3.167 & 1000 & 4.64 & $0.093^{\mathrm{ns}}$ & $-0.970^{\mathrm{ns}}$ & $-1.097^{\mathrm{ns}}$ & $-11.71^{*}$ \\
Total & 34 & 3.022 & 1000 & 6.595 & $0.083^{\mathrm{ns}}$ & $-0.892^{\mathrm{ns}}$ & $-1.127^{\mathrm{ns}}$ & $-24.53^{*}$ \\
\hline
\end{tabular}



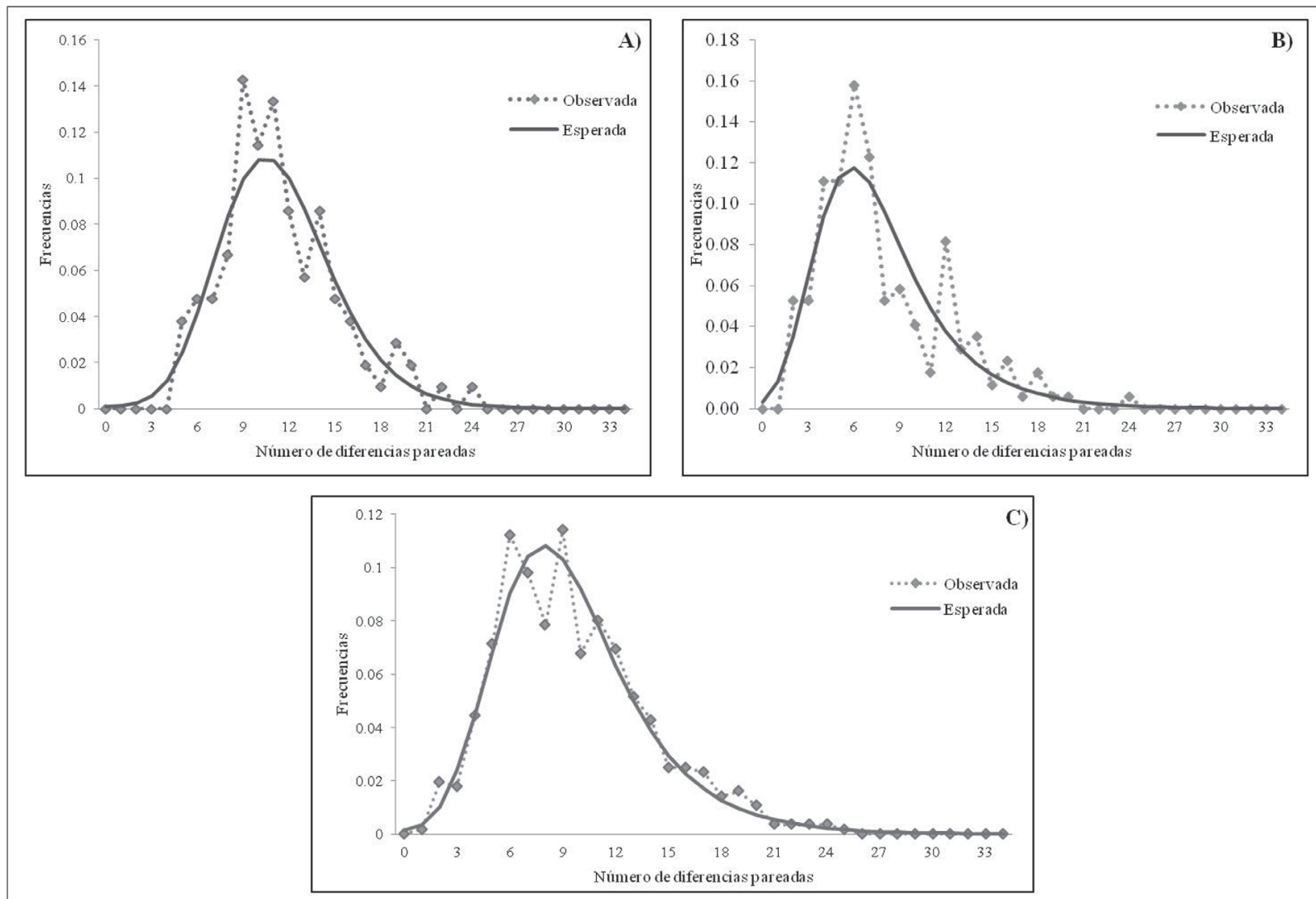

Figura 2. Distribución mismatch de los haplotipos de Artibeus jamaicensis de dos hábitats contrastantes en Chiapas (México), sobre un modelo de expansión poblacional. La línea sólida indica la distribución esperada y la punteada la observada. A) Localidad Pantaleón, B) Localidad Manacal, y C) Total de los individuos.

en la conectividad genética, a escala geográfica fina, se ha evaluado también en otras especies frugívoras y de hábitos generalistas, como Artibeus watsoni, mostrando una $h$ de 0.90 a 0.99 (Ripperger et al. 2013), y Carollia castanea con una $h$ de 0.86 a 0.97 (Ripperger et al. 2014), valores similares a los reportados en este estudio para $A$. jamaicensis. Es posible que la tolerancia a los hábitats perturbados y la alta movilidad de estos murciélagos, los hace menos susceptibles a la fragmentación del hábitat, y les permiten mantener el intercambio genético entre las poblaciones, en comparación con otros murciélagos (Ripperger et al. 2013; Ripperger et al. 2014).

En el presente estudio se encontró un haplotipo por individuo en ambas poblaciones, lo cual se refleja en su diversidad muy alta $(h=1)$. Esto podría deberse al tamaño del muestreo (34 individuos), el cual es pequeño comparado con otros estudios (de 49 a 193 individuos; Carstens et al. 2004; Fleming et al. 2009; Ruíz et al. 2013; Del Real-Monroy et al. 2014), y a la ubicación de las redes de colecta en sitios con disponibilidad de alimentos. Además, el D-loop tiene una alta tasa de evolución, de sustitución y polimorfismo (Lunt et al. 1998), que promueve altos valores de diversidad genética.

Los bajos valores de diversidad nucleotídica $(\pi<0.03)$ reportados en este estudio son congruentes con lo registrado en otros estudios de A. jamaicensis, los cuales oscilan entre 0.009 y 0.1086 (Carstens et al. 2004; Fleming et al. 2009; Ruíz et al. 2013; Del Real-Monroy et al. 2014). La diversidad nucleotídica de la localidad Pantaleón $(\pi=$ $0.029)$ es ligeramente mayor a la de Manacal $(\pi=0.020)$, debido a un mayor número de sitios polimórficos en Pantaleón y a las pocas mutaciones presentes en diferentes sitios polimórficos. Una alta diversidad haplotípica acompañada de una baja diversidad nucleotídica, significa que los haplotipos difieren en un número reducido de nucleótidos, lo cual es característico de efectos de expansión demográfica poblacional en un tiempo relativamente reciente (Hamilton 2009). 


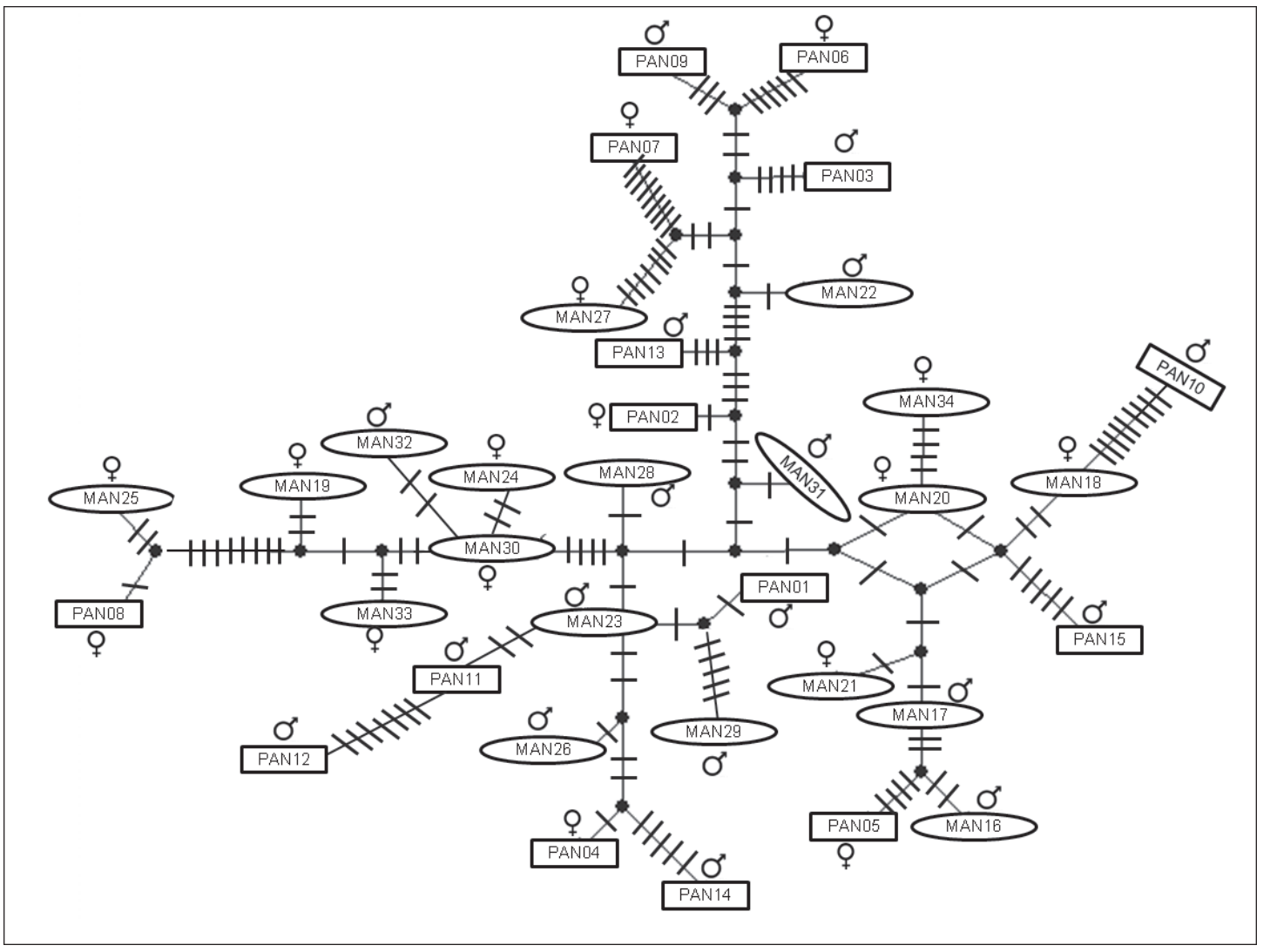

Figura 3. Red de haplotipos de Artibeus jamaicensis de dos hábitats contrastantes en Chiapas (México), construida en Network versión 4.6.1.3, con el método de máxima parsimonia usando un segmento de 396 pb de la región mitocondrial D-loop. Los rectángulos representan los haplotipos de la localidad Pantaleón (Pan), y las elipses representan los haplotipos de la localidad Manacal (Man). Los puntos negros representan un haplotipo hipotético ancestral y las líneas representan los pasos mutacionales $($ hembras $=q ;$ machos $=\widehat{\jmath})$.

La localidad Pantaleón comprende una amplia extensión de vegetación densa y continua, donde se observaron especies raras de murciélagos, vulnerables a la perturbación de su hábitat y reportadas en bosques maduros y densos como Centurio senex Gray, 1842 (Chiroptera: Phyllostomidae), Lonchorhina aurita Tomes, 1863 (Chiroptera: Phyllostomidae) y Rhogeessa sp. (Chiroptera: Vespertilionidae; Schulze et al. 2000; Gómez-Nísino 2006; Ávila-Cabadilla et al. 2009). La cantidad de árboles y la variedad de especies florísticas que conforman esta vegetación, podrían proporcionar mayor cantidad y calidad de refugios, y mayor disponibilidad de alimento para A. jamaicensis en comparación con lo que ofrece la poca cobertura vegetal de la localidad Manacal, la cual es un fragmento de vegetación aislado, resultado de la ganadería extensiva de la zona. No obstante, Manacal alberga árboles ornamentales y frutales, como la almendra (Terminalia catappa Linneo, 1767) y el mango (Mangifera indica
Linneo, 1876); de los cuales sus frutos son frecuentemente consumidos por A. jamaicensis (Kunz \& Díaz 1995). Por lo tanto, ambas localidades proporcionan los recursos necesarios para la persistencia de estos murciélagos, lo cual mantiene sus altos niveles de diversidad genética.

Los recursos que ofrece Manacal para A. jamaicensis son suficientes para que sus individuos no busquen recursos en otros sitios lejanos como Pantaleón. Cuando estos murciélagos tienen refugios con condiciones adecuadas para alimentarse, y tener a sus crías, se mueven a cortas distancias (Ortega et al. 2008). Si hay alimento se mueven máximo a $600 \mathrm{~m}$ de los refugios, pero en hábitats con poca disponibilidad de alimento pueden volar hasta $8 \mathrm{~km}$ por noche (Morrison 1978; Ortega \& Castro-Arellano 2001; Ortega et al. 2002). Aunque algunos estudios genéticos sugieren que la distancia geográfica parece no influir en la estructura genética de $A$. jamaicensis (Vargas-Miranda 2009; Vázquez-Domínguez et al. 2013), la distancia geo- 
gráfica entre Pantaleón y Manacal es suficiente para dar lugar a cierta estructura genética de $A$. jamaicensis, como se ha reportado en zonas núcleo de la Reserva de la Biosfera Maya en Petén, Guatemala (Landaverde-González et al. 2012).

No obstante, existen otros factores que contribuyen o facilitan el flujo génico entre poblaciones de A. jamaicensis en Pantaleón y Manacal, como la dinámica en sus relaciones sociales bajo el sistema de apareamiento poligínico, donde la reproducción no se restringe a las cópulas entre miembros del mismo grupo social (Clutton-Broock 1988), y los individuos se mueven constante y temporalmente entre los grupos sociales dentro de una misma población o entre diferentes poblaciones (Ortega et al. 2004). La aclimatación a distintos tipos de hábitats (Clutton-Broock 1988), y la alta vagilidad de A. jamaicensis, le facilita un flujo génico alto entre sus poblaciones, lo que reduce su diferenciación genética (Carstens et al. 2004; Rossiter et al. 2012; Vázquez-Domínguez et al. 2013; Del Real-Monroy et al. 2014). La conexión entre las poblaciones de A. jamaicensis es sostenida por el resultado del AMOVA, el cual indica que el mayor porcentaje de variación genética se reparte entre los individuos dentro de las poblaciones (94.62\%).

El análisis de las distribuciones mismatch muestra para ambas localidades y en total, curvas multimodales, las cuales sugieren poblaciones en equilibrio demográfico. Sin embargo, la forma de la distribución no es suficiente para rechazar la hipótesis nula de expansión demográfica poblacional relativamente reciente (Yun-Dong et al. 2015). Un proceso de expansión poblacional reciente produce un patrón unimodal sesgado hacia la izquierda de la distribución mismatch, debido a las comparaciones entre secuencias idénticas o similares (Rogers \& Harpending 1992). Esto es observado en las distribuciones mismatch de ambas localidades, sugiriendo que $A$. jamaicensis experimentó una expansión demográfica poblacional repentina y relativamente reciente en su historia evolutiva, lo cual también es apoyado por la $R_{2} \mathrm{y} \mathrm{SSD}$.

Los valores negativos de las pruebas de neutralidad $\mathrm{D}, \mathrm{D}^{*}$ y $F_{\mathrm{S}}$ para ambas localidades, y en total, también se asocian a una rápida expansión poblacional. Los valores negativos en las pruebas de neutralidad se dan porque los alelos raros son más numerosos de lo esperado, señalando poblaciones que han experimentado una expansión reciente (Ramos-Onsins \& Rozas 2002). Los valores no fueron significativos para D y D*, pero si para $F_{\mathrm{s}}$. De acuerdo a $\mathrm{Fu}(1997)$, la prueba $F_{\mathrm{S}}$ es más potente para detectar crecimiento poblacional y expansión demográfica reciente o selección, que el estadístico D (Tajima 1989), y generalmente arroja grandes valores negativos, como los obtenidos en este estudio.

Otros estudios también han señalado que $A$. jamaicensis experimentó una expansión demográfica repentina y reciente (Carstens et al. 2004; Larsen et al. 2007; Vargas-Miranda 2009; Ruíz et al. 2013), asociado a la glaciación del Pleistoceno (Phillips et al. 1991; Grant \& Bowen 1998). Durante la última máxima glaciación, las regiones templadas fueron cubiertas por grandes extensiones de hielo, obligando a las especies a desplazarse hacia refugios en diferentes áreas geográficas (Hewitt 2000). Se asume que el crecimiento poblacional y la expansión geográfica produjeron, probablemente, una rápida diversificación de haplotipos con mínimas diferencias nucleotídicas (Grant $\&$ Bowen 1998). Es por ello, que se esperan poblaciones actuales con alta diversidad haplotípica y baja diversidad nucleotídica, las cuales están asociados a eventos de crecimiento poblacional y colonización, durante y después, de las glaciaciones del Pleistoceno (Shiang-Fan et al. 2006).

El patrón de diversidad haplotídica y nucleotídia de $A$. jamicensis en Pantaleón y Manacal coincide con lo esperado en poblaciones que han experimentado una reciente expansión demográfica. No obstante, los tiempos de divergencia y expansión inferidos a través de datos moleculares son hipotéticos y los estimadores poseen errores grandes (Hillis et al. 1996). Por otro lado, el tamaño de muestra y poblaciones pueden generar un sesgo en la estimación de parámetros poblacionales, por lo que la expansión demográfica y geográfica reciente de $A$. jamaicensis deber tomarse como una hipótesis que requiere ser sometida a prueba en estudios futuros.

La topología de la red de haplotipos mostrada en el presente estudio, donde a partir de un haplotipo central surgen varios haplotipos separados por un único paso mutacional, también son indicios de una reciente expansión demográfica poblacional (Forster 2004). En la red de haplotipos la distribución geográfica no se relaciona con la distribución de los haplotipos. En el interior como en los extremos de la red, los haplotipos de ambas localidades se mezclan. La ausencia de una división geográfica en la red de haplotipos puede deberse a la escala geográfica fina del estudio y a la alta vagilidad de $A$. jamaicensis. Sin embargo, se ha observado la mezcla de haplotipos entre poblaciones insulares de A. jamaicensis separadas por grandes distancias (> $100 \mathrm{~km}$ de océano; Carstens et al. 2004).

Por tratarse de una especie con una historia evolutiva reciente (Ruíz et al. 2013) hace falta incluir poblaciones 
de diferentes lugares de México, para identificar qué procesos demográficos históricos han dejado marcas evolutivas en la distribución geográfica actual de los haplotipos de $A$. jamaicensis. Para futuros trabajos genéticos se recomienda ampliar el área de estudio, aumentar el tamaño de muestra y el tamaño del fragmento de ADN, así como complementar la información de un gen mitocondrial con un gen nuclear, para tener un mejor panorama de las implicaciones de la modificación del hábitat en la diversidad genética de $A$. jamaicensis.

\section{CONCLUSIONES}

Las poblaciones de $A$.jamaicensis en Manacal y Pantaleón presentan altos niveles de diversidad genética, lo cual es indicio de que los hábitats de ambas localidades ofrecen los recursos necesarios para la permanencia de los individuos dentro de las poblaciones, y para el mantenimiento de su alta diversidad genética. Al tener los recursos necesarios en ambas localidades, da lugar al desplazamiento reducido de estos murciélagos en el área de estudio, lo cual se refleja en cierta diferenciación genética. A pesar de lo anterior, existen otros factores que permiten la conexión e interacción entre ambas poblaciones de $A$. jamaicensis como sus atributos de especie generalista, su dinámica social bajo el sistema poligínico, y su alta capacidad de desplazamiento.

AGRADECIMIENTOS. Agradecemos a la Dra. Consuelo Lorenzo Monterrubio por su valiosa colaboración y apoyo en la realización de este trabajo. Al Dr. Javier Barrientos Villalobos por su colaboración en el análisis de datos. Al Biol. Víctor Hugo Mendoza Sáenz por su apoyo y colaboración en la colecta de muestras. A los propietarios de los predios donde se realizaron las colectas por permitirnos trabajar en los sitios de muestreo. Al apoyo financiero (PAT) de El Colegio de la Frontera Sur (ECOSUR) y la beca CONACyT otorgada al primer autor para finalizar sus estudios de posgrado. Finalmente, agradecemos a los revisores, por sus aportes para mejorar la versión final del manuscrito.

\section{LITERATURA CITADA}

Altschul, S. F., Gish, W., Miller, W., Myers, E. W. \& Lipman, D. J. 1990. Basic local alignment search tool. Journal of Molecular Biology, 215, 403-410.

Ávila-Cabadilla, L. D., Stoner, K. E., Henry, M. \& Alvarez-Añorve, M. Y. 2009. Composition, structure and diversity of phyllostomid bat assemblages in different successional stages of a tropical dry forest. Forest Ecology and Management, 258, 986996.

Bandelt, H. J, Forster, P. \& Röhl, A. 1999. Median-joining networks for inferring intraspecific phylogenies. Molecular Biology Evolution, 16, 37-48.

Bicca-Marques, J. C. 2003. How do howler monkeys cope with habitat fragmentation? pp. 283-30. In: L. K., Marsh (Ed.). Primates in fragments: Ecology and conservation. Kluwer Academic Plenum Publishers. New York.

Carstens, B., Sullivan, J., Davalos, L. M., Larsen, P. A. \& Pedersen, S. C. 2004. Exploring population genetic structure in three species of Lesser Antillean bats. Molecular Ecology, 13, 2557-2566.

Castro-Luna, A. A. 2007. Cambios en los ensamblajes de murciélagos en un paisaje modificado por actividades humanas en el trópico húmedo de México. Tesis de doctorado. Instituto de Ecología, A. C. Xalapa, Veracruz, 119 pp.

Clutton-Brock, T. H. 1988. Reproductive success. pp. 472-486. In: T. H. Clutton-Brock (Ed.). Reproductive Success. The University of Chicago Press, Chicago, Illinois.

Covaleda, S., Aguilar, S., Ranero, A., Marín, I. \& Paz, F. 2014. Diagnóstico sobre determinantes de deforestación en Chiapas. Alianza México para la reducción de emisiones por deforestación y degradación. México, D. F., 150 pp.

Del Real-Monroy, M., Martínez-Méndez, N. \& Ortega, J. 2014. MHC-DRB exon 2 diversity of the Jamaican fruit-eating bat ( $\mathrm{Ar}$ tibeus jamaicensis) from Mexico. Acta Chiropterologica, 16, 301314.

Eisenberg, J. F. \& Redford, K. H. 1999. Mammals of the Neotropics. The central Neotropics: Ecuador, Perú, Bolivia, Brazil: The University of Chicago Press, Chicago, 593 pp.

Excoffier, L., Laval, G. \& Schneider, S. 2006. Arlequin version 3.1: an integrated software package for population genetics data analysis. Evolutionary Bioinformatics, 1, 47-50. Available at: http://cmpg.unibe.ch/software/arlequin3/

Fahrig, L. 2003. Effects of habitat fragmentation on biodiversity. Annual Review of Ecology, Evolution, and Systematics, 34, 487-515.

FAO 2010. Organización de las Naciones Unidas para la Agricultura y la Alimentación. Evaluación de los recursos forestales mundiales 2010. Informe Nacional México. Roma, Italia.

Fenton, M., Acharya, L., Audet, D., Hickey, M., Merriam, C., Obrist, M., Syme, D. \& Adkins, B. 1992. Phyllostomid bats (Chiroptera: Phyllostomidae) as indicators of habitat disruption in the Neotropics. Biotropica, 24, 440-446.

Fleming, T. H., Murray, K. L. \& Carstens, B. 2009. Phylogeography and genetic structure of three evolutionary lineages of West Indian Phyllostomidae bats. pp. 116-150. In: T. H. Fleming \& P.A. Racey (Eds.). Island bats: evolution, ecology and conservation. The University of Chicago Press. Chicago.

Fluxus Technology Ltd. 2012. Network 4.6.1.3. Available at: http:// www.fluxus-engineering.com/sharenet.htm\#ug

Forster, P. 2004. Ice Ages and the mitochondrial DNA chronology of human dispersals: a review. Philosophical Transactions of the Royal Society of London. Series B: Biological Sciences, 359, $255-$ 264.

Fu, Y. X. \& Li, W. H. 1993. Statistical tests of neutrality of mutations. Genetics, 133, 693-709.

Fu, Y. X. 1997. Statistical tests of neutrality of mutations against po- 
pulation growth, hitchhiking and backgroud selection. Genetics, 147, 915-925.

Galindo-González, J. 2004. Clasificación de los murciélagos de la región de los Tuxtlas, Veracruz, respecto a su respuesta a la fragmentación del hábitat. Acta Zoológica Mexicana, 20, 239-243.

Galindo-González, J. 2007. Efectos de la fragmentación del paisaje sobre las poblaciones de mamíferos, el caso de los murciélagos de Los Tuxtlas Veracruz. Pp. 97-114. In: G. Sánchez-Rojas \& A. E. Rojas-Martínez (Eds.). Tópicos en Sistemática, Biogeografía, Ecología y Conservación de Mamíferos. Universidad Autónoma del Estado de Hidalgo, México.

Gómez-Nísino, A. 2006. Ficha técnica de Lonchorhina aurita. In: R. Medellín (Ed.). Los mamíferos mexicanos en riesgo de extinción según el PROY-NOM-059-ECOL-2000. Instituto de Ecología, Universidad Nacional Autónoma de México, D. F.

Grant, W. S. \& Bowen, B. W. 1998. Shallow population histories in deep evolutionary lineages of marine fishes: insights from sardines and anchovies and lesson for conservation. The Journal of Heredity, 89, 415-426.

Hamilton, M. B. 2009. Populations genetics. Wiley-Blackwell, Oxford, $424 \mathrm{pp}$.

Hewitt, G. M. 2000. The genetic legacy of the Ice Ages. Nature, 405, 907-913.

Hillis, D. M., Moritz, C. \& Mable, B. K. 1996. Applications of molecular systematics: the state of the field and a look to the future. pp. 515-543. In: D. M. Hillis, C. Moritz \& B. K. Mable (Eds.). Molecular Systematics, Sinauer Associates, Sunderland, Massachusetts.

Holderegger, R. \& Di Giulio, M. 2010. The genetic effects of roads: a review of empirical evidence. Basic and Applied Ecology, 11, $522-531$.

Hudson, R. R., Boos, D. D. \& Kaplan, N. L. 1992. A statistical test for detecting population subdivision. Molecular Biology Evolution, 9, 138-151.

Jones, K. E., Barlow, K. E., Vaughan, N., Rodríguez-Durán, A. \& Gannon, M. R. 2001. Short-term impacts of extreme environmental disturbance on the bats of Puerto Rico. Animal Conservation, 4, 59-66.

Juste, J., Bilgin, R., Muñoz, J. \& Ibáñez, C. 2009. Mitochondrial DNA signatures at different spatial scales: from the effects of the Straits of Gibraltar to population structure in the meridional serotine bat (Eptesicus isabellinus). Heredity, 103, 178-187.

Kaplan, N. L., Darden, T. \& Hudson, R. 1989. The coalescent process in models with selection. Genetics, 120, 819-829.

Kimura, M. 1991. The neutral theory of molecular evolution: A review of recent evidence. Japanese Journal of Genetics, 66, 367386.

Kunz, T. H., \& Díaz, C. A. 1995. Folivory in fruit-eating bats with new evidence from Artibeus jamaicensis (Chiroptera: Phyllostomidae). Biotropica, 27, 106-120.

Kunz, T. H., Arnett, E. B., Erickson, W. P., Hoar, A. R., Johnson, G. D., Larkin, R. P., Strickland, M. D., Threshe, R. W. \& Tuttle, M. D. 2007. Ecological impacts of wind energy development on bats: questions, research needs, and hypotheses. Frontiers in Ecology and the Environment, 5, 315-324.

Landaverde-González, P., Calderón, A. P., Solórzano, E. \& Ariza, M. A. 2012. Efecto de la fragmentación sobre el flujo génico de
Artibeus jamaicensis en el Biotopo el Zotz y su zona de amortiguamiento en Petén, Guatemala. Universidad de San Carlos de Guatemala. Informe técnico.

Larkin, M. A., Blackshields, G., Brown, N. P., Chenna, R., Mcgettigan, P. A., Mcwilliam, H., Valentin, F., Wallace, I. M., Wilm, A., Lopez, R., Thompson, J. D., Gibson, T. J. \& Higgins, D. G. 2007. Clustal X Version 2.0. Bioinformatics, 23, 2947-2948.

Larsen, P. A., Hoofer, S. R., Bozeman, M. C., Pedersen, S. C., Genoways, H. H., Phillips, C. J., Pumo, D. E. \& Baker, R. J. 2007. Phylogenetics and phylogeography of the Artibeus jamaicensis complex based on cytochrome-b DNA sequences. Journal of Mammalogy, 88, 712-727.

Librado, P. \& Rosas. J. 2009. DnaSP version 5: a software for comprehensive analysis of DNA polymorphism data. Bioinformatics, 25, 1451-1452.

Lim, B. K., Engstrom, M. D., Lee, T. E., Patton, J. C. \& Bickham, J. W. 2004. Molecular differentiation of large species of fruit-eating bats (Artibeus) and phylogenetic relationships based on the cytochrome b gene. Acta Chiropterologica, 6, 1-12.

Lunt, D. H., Whippl, L. E. \&. Hyman, B. C. 1998. Mitochondrial DNA variable number tandem repeats (VNTRs): utility and problems in molecular ecology. Molecular Ecology, 7, 1441-1455.

Medellín, R. A., Arita, H. \& Sánchez, O. 2008. Identificación de los murciélagos de México, clave de campo. Instituto de Ecología, UNAM, México, D. F., 79 pp.

Melo, F. P. L., Rodríguez-Herrera, B., Chazdon, R. L., Medellín, R. A. \& Ceballos, G. G. 2009. Small tent-roosting bats promote dispersal of large-seeded plants in a Neotropical forest. Biotropica, 41, 737-743.

Meyer, C. F. J., Kalko, E. K.V. \& Kerth, G. 2009. Small-scale fragmentation effects on local genetic diversity in two phyllostomid bats with different dispersal abilities in Panama. Biotropica, 41, 95-102.

Miller-Butterworth, C. M., Jacobs, D. S. \& Harley, E. H. 2003. Strong population substructure is correlated with morphology and ecology in a migratory bat. Nature, 424, 187-191.

Mittermeier, R. A., Robles, P. \& Goettsch, C. 1997. Megadiversidad. Los países biológicamente más ricos del mundo. México, D. F., $76 \mathrm{pp}$.

Morrison, D. W. 1978. Foraging ecology and energetics of the frugivorous bat Artibeus jamaicensis. Ecology, 59, 716-723.

Nei, M. 1987. Molecular Evolutionary Genetics. Columbia University Press, New York, 512 pp.

Ortega, J. \& Arita, H. T. 1999. Structural and social dynamics of harem groups in Artibeus jamaicensis (Chiroptera: Phyllostomidae). Journal of Mammalogy, 80, 1173-1185.

Ortega, J. \& Arita, H. T. 2004. Estructura social y movimientos de los murciélagos zapoteros (Artibeus jamaicensis) en un ambiente poligínico. pp. 363-374. In: V. Sánchez-Cordero \& R. A. Medellín (Eds.). Contribuciones mastozoológicas en homenaje a Bernardo Villa. Instituto de Biología, UNAM, México, D. F.

Ortega, J. \& Castro-Arellano, I. 2001. Artibeus jamaicensis. Mammalian Species, 662, 1-9.

Ortega, J., Guerrero, J. A. \& Maldonado, J. E. 2008. Aggression and tolerance by dominant males of Artibeus jamaicensis: strategies to maximize fitness in harem groups. Journal of Mammalogy, $89,1372-1378$. 
Ortega, J., Maldonado, E., Arita, H., Wilkinson, G. \& Fleischer, R. 2002. Characterization of microsatellite loci in the Jamaican fruiteating bat Artibeus jamaicensis and cross species amplification. Molecular Ecology Notes, 2, 462-464.

Ortega, J., Maldonado, J. E., Wilkinson, G. S., Arita, H. T. \& Fleischer, R. C. 2003. Male dominance, paternity and relatedness in the Jamaican fruit-eating bat (Artibeus jamaicensis). Molecular Ecology, 12, 2409-2415.

Perfectti, F., Picó, F. X. \& Gómez, J. M. 2009. La huella genética de la selección natural. Ecosistemas, 18, 10-16.

Peters, S. L., Malcolm, J. R. \& Zimmerman, B. L. 2006. Effects of selective logging on bat communities in Southeastern Amazon. Conservation Biology, 20, 1410-1421.

Phillips, C. J., Pumo, D. E., Genoways, H. H., Ray, P. E. \& Briskey, C. A. 1991. Mitocondrial DNA evolution and phylogeography in two Neotropical Fruit Bats Artibeus jamaicensis and Artibeus lituratus. pp. 97-123. In: M. A. Mares \& D. J. Schmidly (Eds.). Latin American mammalogy, history, biodiversity and conservation. University of Oklahoma Press.

Pumo, D. E., Kim, I., Remsen, J., Phillips, C. \& Genoways, H. H. 1996. Molecular systematics of the fruit bat, Artibeus jamaicensis: origin of an unusual island population. Journal of Mammalogy, 77, 491-503.

Ramos-Onsins, S. E. \& Rozas, J. 2002. Statistical properties of new neutrality tests against population growth. Molecular Biology and Evolution, 19, 2092-2100.

Reid, F.A. 2009. A Field Guide to the Mammals of Central America \& Southeast Mexico. Oxford University Press, New York, 384 pp.

Ripperger, S. P., Tschapkab, M., Kalko, E. K., Rodríguez-Herrera, B. \& Mayer, F. 2013. Life in a mosaic landscape: anthropogenic habitat fragmentation affects genetic population structure in a frugivorous bat species. Conservations Genetics, 14, 925-934.

Ripperger, S. P., Tschapkab, M., Kalko, E. K., Rodríguez-Herrera, B. \& Mayer, F. 2014. Resisting habitat fragmentation: high genetic connectivity among populations of the frugivorous bat Carollia castanea in an agricultural landscape. Ecosystems and Environment, 185, 9-15.

Rogers, A. R. \& Harpending, H. 1992. Population growth makes waves in the distribution of pairwise genetic differences. Molecular Biology and Evolution, 9, 552-569.

Rossiter, S. J., Jones, G., Ransome, R. \& Barratt, E. M. 2000. Genetic variation and population structure in the endangered greater horseshoe bat Rhinolophus ferrumequinum. Molecular Ecology, 9, 1131-1135.

Rossiter, S. J., Zubaid, A., Mohd-Adnan, A., Struebig, M. J., Kunz, T. H., Gopal, S., Petit, E. J. \& Kingston, T. 2012. Social organization and genetic structure: insights from codistributed bat populations. Molecular Ecology, 21, 647-661.

Ruíz, E., Vargas-Miranda, B. \& Zúñiga, G. 2013. Late-Pleistocene phylogeography and demographic history of two evolutionary lineages of Artibeus jamaicensis (Chiroptera: Phyllostomidae) in Mexico. Acta Chiropterologica, 15, 19-33.

Sambrook, J., Fritsh, E. F. \& Maniatis, T. 1989. Molecular Cloning: A Laboratory Manual. Cold Spring Harbor Laboratory Press, New York, $1626 \mathrm{pp}$.

Sánchez, R. 2009. Cambios en la estructura de la comunidad de murciélagos de la Estación Biológica La Selva, Costa Rica: 1973 y
2005. Tesis de Maestría. Facultad de Ciencias, UNAM. México.

Saunders, D. A. Hobbs, R. J. \& Margules, C. R. 1991. Biological consequences of ecosystem fragmentation: a review. Conservation Biology, 5, 18-32.

Schulze, M. D., Seavy, N. E. \& Whitacre, D. F. 2000. A comparison of phyllostomid bat assemblages in undisturbed Neotropical forest and in forest fragments of a slash and burn farming mosaic in Petén, Guatemala. Biotropica, 32, 174-184.

Shiang-Fan, C., Rossiter, S. J., Faulkes, C. G. \& Jones, G. 2006. Population genetics structure and demographic history of the endemic Formosan lesser horseshoe bat (Rhinolophus monoceros). Molecular Ecology, 15, 1643-1656.

Slatkin, M. \& Hudson, R. R. 1991. Pairwise comparisons of mitochondrial DNA sequences in stable and exponentially growing populations. Genetics, 129, 555-562.

Soriano, P. J. \& Ochoa, J. G. 2001. The consequences of timber exploitation for bat communities in tropical America. pp. 153-166. In: R. A. Fimbel, A. Grajal, J. G. Robinson (Eds.). The Cutting Edge: Conserving Wildlife in Logged Tropical Forests. Columbia University Press, New York, N.Y.

Studier, E. H., Boyd, B. C., Felman, A. T., Dapson, R. \& Wilson, D. E. 1983. Renal function in the Neotropical bat, Artibeus jamaicensis. Comparative Biochemistry and Physiology part a Physiology, 74, 199-209.

Tajima, F. 1989. Statistical method for testing the neutral mutation hypothesis by DNA polymorphism. Genetics, 123, 585-595.

Technelysium Pty Ltd. 2003-2009. ChromasPro version 1.5. Available at: http://softadvice.informer.com/Chromaspro_Download. html

Vargas-Miranda, B. 2009. Filogeografía de Artibeus jamaicensis triomylus y Artibeus jamaicensis yucatanicus (Chiroptera: Phyllostomidae) de México. Tesis de doctorado. Escuela Nacional de Ciencias Biológicas, Instituto Politécnico Nacional. México, D. F. 92 pp.

Vázquez-Domínguez, E., Mendoza-Martínez, A., Orozco-Lugo, L. \& Cuarón, A. D. 2013. High dispersal and generalist habits of the bat Artibeus jamaicensis on Cozumel Island, Mexico: an assessment using molecular genetics. Acta Chiropterologica, 15, 411-421.

Wilcove, D. S., McLellan, C. H. \& Dobson, A. P. 1986. Habitat fragmentation in the temperate zone. pp. 237-56. In: M. E. Soulé (Ed.). Conservation Biology. Sunderland, Sinauer.

Wilcox, B. A. \& Murphy, D. D. 1985. Conservation strategy: the effects of fragmentation on extinction. The American Naturalist, $125,879-887$.

Wilkinson, G. S. \& Chapman, A. M. 1991. Length and sequence variation in evening bat D-loop mtDNA. Genetics, 128, 607-617.

Wilkinson, G. S., Mayer, F., Kerth, G. \& Petri, B. 1997. Evolution of repeated sequence arrays in the D-loop region of bat mitochondrial DNA. Genetics, 146, 1035-1048.

Wright, S. 1951. The genetical structure of populations. Annals of Eugenics, 15, 323-354.

Yun-Dong, G., Yu, Z., Xin-Fen, Z. \& Zhang-Ming, Z. 2015. Pleistocene glaciations, demographic expansion and subsequent isolation promoted morphological heterogeneity: A phylogeographic study of the alpine Rosa sericea complex (Rosaceae). Scientific Reports, 5, 11-98. 